TAIWANESE JOURNAL OF MATHEMATICS

Vol. 4, No. 1, pp. 9-20, March 2000

\title{
ON SHOCK WAVE THEORY
}

\author{
Tai-Ping Liu* \\ Dedicated to Fon-Che Liu on his sixtieth birthday
}

\begin{abstract}
We survey the qualitative shock wave theory. The survey is meant to explain some of the key issues close to the interest of the author and raise open questions.
\end{abstract}

\section{Hyperbolic Conservation Laws}

The simplest equations to describe the shock waves are the hyperbolic conservation laws

$$
u_{t}+f(u) x=0 .
$$

The theory started with the investigation of the Euler equations in the gas dynamics

$$
\rho_{t}+(\rho v)_{x}=0,(\rho v)_{t}+\left(\rho v^{2}+p\right)_{x}=0,(\rho E)_{t}+(\rho E v+p v)_{x}=0 .
$$

The book Courant-Friedrichs [11] gives the acount of the efforts on the equations by many of the leading mathematicians before 1948 .

Starting about the '40s, it was realized that it is possible to describe some of the key features of the shock behaviour by the scalar equation. The mathematical theory of well-posedness for scalar equation, $u \in R^{1}$, even for several space dimension $x \in R^{n}$, is now well-understood; cf. Krushkov [21]. The theory for scalar law has been generalized to Hamilton-Jacobi equation. One of the interesting properties of the solution operator is the compactness property.

Received December 27, 1999.

Communicated by P. Y. Wu.

2000 Mathematics Subject Classification: 35L67, 76L05, 35L65, 35L05.

Key words and phrases: Shock wave, conservation law.

${ }^{*}$ Research supported in part by NSF Grant DMS-9803323. 
For instance, for convex law, $f^{\prime \prime}(u) \neq 0$, there are two time-invariants and that the oscillation of the initial data is immediately damped; cf. Lax [24]. This inviscid dissipation is still conjectured for the incompressible flow and easily understood for scalar conservation law. It sets apart the shock wave theory from other nonlinear wave theories.

Two important elements of the theory for hyperbolic conservation laws are the entropy condition for shock waves and the compactness property of the solution operator due to the nonlinearity of the flux function $f(u)$. The study of the consequences of these, particularly on the qualitative behaviour of the solutions, is the goal of the theory for hyperbolic conservation laws. There is the notion of genuine nonlinearity and the entropy condition of Lax [23] which are the generalization of the convexity of the scalar flux $f^{\prime \prime}(u) \neq 0$ and the compressibility of the shocks.

For systems of conservation laws, $u \in R^{n}, n \geq 2$, there are rich wave interaction phenomena. The classical works of Glimm [17], Glimm-Lax [18] study the nonlinear wave interactions and qualitative behavior of the solutions. There are several subsequent works: DiPerna [12,13], and Liu [28, 29,31] on regularity and large-time behavior. There is the wave tracing technique (Liu [30]), which yields the more definite form of the principle of nonlinear superposition and is useful for the study of solution behaviour.

For systems which are not necessarily genuinely nonlinear, there is the entropy condition of Liu [27]. The qualitative behaviour of the solutions is richer and more complex; see Liu [31], Cheng [9] and Chern [10].

There are recent important developments in the well-posedness theory. One theory is based on Bressan [1] on the $L_{1}$-stability for infinitesimal variation of the initial data; cf. Bressan-Crasta-Picolli [2]. The other is to construct a time-decreasing nonlinear functional which is equivalent to the $L_{1}$-distance of two solutions (Liu-Yang $[38,39]$ ). One can formulate as an easy consequence of the well-posedness theory the function class for which the uniqueness theory holds; cf. [3].

The well-posedness theory is based on Glimm's construction of solutions and the study of nonlinear wave interactions (Glimm [17]). Through the wave tracing mechanism (Liu [31]), the construction of the nonlinear functional (Liu-Yang [39]) is reduced to the study of the effects of wave coupling between the solutions on their $L_{1}$-distance. Central to this study is the introduction of a generalized entropy functional (Liu-Yang [38]). The generalized entropy functional makes effective usage of the nonlinearity of the flux and is new even for the basic inviscid Burgers equation. The idea in Liu-Yang [39] complements and is orthogonal to the classical work of Glimm [17] on nonlinear wave interactions in a given solution. 
So far there are two theories for hyperbolic conservation laws. There is the aforementioned qualitative theory originated with Glimm's seminal works and followed up with the recent well-posedness theory. The other theory is the existence theory base on the theory of compensated compactness, starting with the work of Tartar [49]. There have been intensive activities on the existence theory for two conservation laws and some works on large-time behaviors; cf. [7] and references therein. The solutions obtained by this approach of compensated compactness have not been shown to possess the nonlinear wave behavior. As a consequence, the aforementioned well-posedness theory is not applicable to these solutions.

A fundamental open problem is to study the qualitative behavior of a general weak solution, not necessarily the one constructed by a particular method. So far, it is not known, for instance, if a weak solution satisfies such a basic property of hyperbolic equations as the finite speed of propagation. In fact, the verification of this would lead to the understanding of the nonlinear wave interaction property. Ultimately, one hopes to study the wave behaviors, such as local regularity (cf. [13,31]), and large-time behavior $([28,29])$ of the solutions. Sufficient regularity of the solutions would allow the application of the aforementioned well-posedness theory.

The compactness theory of Glimm-Lax [18] applies to $2 \times 2$ systems. It says that solutions with small sup norm but infinite variation norm initial data become of local bounded variation for any positive time. This is easily seen for scalar equations; cf. Lax [24]. For $2 \times 2$ systems, wave interactions are of third order due to the existence of the coordinates of Riemann invariants. This makes it possible to generalize the scalar compactness result to $2 \times 2$ systems. It is not known whether such a strong compactness result holds for more general systems, such as the full $3 \times 3$ gas dynamics equations.

\section{Viscous Conservation LaWs}

That the role of viscosity is important has been recognized since the time of Stokes. It is essential in the discussion of the entropy condition for shock waves. Consider the viscous conservation laws

$$
u_{t}+f(u)_{x}=\left(B(u, \varepsilon) u_{x}\right)_{x},
$$

where $B(u, \varepsilon)$ is the viscosity matrix. An important example is the NavierStokes equations in gas dynamics

$\rho_{t}+(\rho v)_{x}=0,(\rho v)_{t}=\left(\rho v^{2}+p\right)_{x}=\left(\mu v_{x}\right)_{x},(\rho E)_{t}+(\rho E v+p v)_{x}=\left(\mu v v_{x}+\kappa T_{x}\right)_{x}$.

The dissipation parameters $\varepsilon$ here are the viscosity $\mu$ and the heat conductivity $\kappa$. When the dissipation parameters $\varepsilon$ are turned off, the inviscid system 
becomes the hyperbolic conservation laws

$$
B(u, 0)=0 .
$$

When the hyperbolic conservation law is strictly hyperbolic, such as the Euler equations, a shock $\left(u_{-}, u_{+}\right)$satisfies the entropy condition if and only if it is the limit of viscous traveling waves

$$
u(x, t)=\varphi(x-s t), \quad \varphi( \pm \infty)=u_{ \pm} .
$$

For non-strictly hyperbolic conservation laws, the situation is more complicated and interesting, as we will see later. We mention here that vacuum in the gas flow gives rise to singularities different from the shocks. In fact, shocks vanish upon hitting the vacuum [36]. For the interesting and important studies of the question of vacuum, see the article of Tong Yang in this issue.

There has been a substantial progress on the stability of viscous waves for systems with artificial viscosity

$$
u_{t}+f(u)_{x}=\varepsilon u_{x x}
$$

cf. Liu [35], Yu [52], Zumbrun-Howard [55] and the references therein. The main technique is the pointwise estimate, which yields the solution behavior on and off characteristic directions. It originates from the study of the perturbation of a constant state; see Liu [33]. The study of viscous contact continuities remains largely open, though there is the preliminary work of LiuXin [37]. In a forthcoming paper, Yu and the author have succeeded in solving the Riemann problem (Liu-Yu [42]).

For hyperbolic conservation laws, the Riemann problem

$$
u(x, 0)= \begin{cases}u_{\ell} & , \quad x<0 \\ u_{r} & , \quad x>0\end{cases}
$$

has the simple scaling and the solution is a function of $x / t, u(x, t)=\varphi(x / t)$. The viscous conservation laws do not have such simple scaling. For small time, the scaling is that of linear parabolic equations. The intermediate time is characterized by the hyperbolic scaling. The large-time contains a subtle logarithmic scaling as the result of interaction of rarefaction and compression waves. This simple problem thus yields rich wave phenomena. By simple scaling, the large-time behaviour of the Riemann solution is also the zero dissipation limit. The zero dissipation limit is an important problem and has been studied only for local time when the shocks are isolated (Goodman-Xin [19], $\mathrm{Yu}[52])$. It is important to consider the solution of the viscous conservation 
laws when the dissipation $\varepsilon$ is nonzero but small. The compensated compactness approach yields zero dissipation limit for particular $2 \times 2$ systems, though without qualitative understanding of the solutions.

For physical systems, the viscosity matrix $B(u, \varepsilon)$ is in general degenerate and the viscous conservation laws are not uniformly parabolic, but hyperbolicparabolic. There is the basic energy method for the dissipation about a constant state (Kawashima [20]). One interesting consequence of the hyperbolicparabolic nature of the system is that the Green function for the linearized system contains $\delta$-functions. This and the pointwise study of the diffusion waves can be found in Liu-Zeng [43]. Much remains to be done for the study of nonlinear waves for the physical viscous conservation laws.

The situation becomes more complicated when the inviscid system is not strictly hyperbolic. An important case concerns the intermediate shocks for magneto-hydrodynamics; cf. Freistuhler-Liu [15]. There is also the example of combustions. Waves which are more compressive than the classical gas dynamics shocks are physical only as viscous waves and not as inviscid waves. Thus the intermediate shocks are stable only if the perturbation is small compared to the strength of the dissipations. Waves less compressive depend, in both thier existence and structure, sensitively on the form of dissipations. For instance, the weak detonations and deflagrations in the combustions cannot be described by the inviscid theory of Chapman-Jouget; cf. [11]. For these nonclassical shocks, see Liu [34] and the references therein. So far these waves are studied only for simplified systems and their study for the whole physical systems remains to be done.

\section{Shock and Reaction-Diffusion Waves}

Shock waves are one kind of nonlinear waves. Two other important classes of nonlinear waves are dispersion waves and reaction-diffusion waves. It is, of course, important to relate these distinct classes because a general physical situation often exhibits waves of mixed types. For the study of the limit of the dispersve equations to hyperbolic conservation laws, see Lax-LevermoreVenakidis [22]. We now illustrate with an important physical situation of combustions, which relate the shock waves with reaction-diffusion waves.

There are two classes of combustion waves in gas: the high-speed detonation waves and low-speed deflagration waves. The strong detonation wave is led by a gas dynamics shock, which raises the temperature beyond the ignition point, and is then followed by a reaction zone. Thus the strong detonation wave is dominated by the shock wave. The weak detonation wave is supersonic and precedes gas dynamics waves. Thus it is a reaction-diffusion wave. The slow-speed weak deflagration wave is usually studied by the reaction-diffusion 
equations. This is done by assuming that the underlying gas flow is given and not affected by the reaction.

To study the combustion waves in gas, there is the classical ChapmanJouget inviscid theory (Courant-Friedrichs [11]). The theory is adequate for the strong detonations. To study the weak detonations and weak deflagrations, the role of diffusions becomes important. In fact, the existence of these waves depends sensitively on the dissipation parameters, a common feature of undercompression waves [34]. There is analysis of weak detonations for a simple model of Rosales-Majda [46] in Liu-Yu [41]. As indicated above, the analysis necessarily contains thinking of reaction-diffusion waves. The chemical nonlinearity is more important than the gas dynamics nonlinearity for the stability analysis.

The simplest realistic physical model for the gas combustions is the reactive Navier-Stokes equations. It would be interesting to study the stability of weak detonations and weak deflagrations for the reactive Navier-Stokes equations. Besides the reaction- diffusion nature of these waves, one needs also to consider the interactions with the gas dynamics waves as mentioned in the last section. The combustion waves for the reactive Navier-Stokes equations have been constructed in Gasser-Szmolyan [16].

\section{Conservation Laws and Dynamics Systems}

The rich theory for ODE, e.g., KAM theory and chaos, has begun to have impact on nonlinear PDEs. There are, for instance, works on semilinear wave equations. For conservation laws, there are interesting works for scalar conservation laws using the ideas from dynamics systems, e.g., [14, 47]. One property of the scalar equation, which is essential for these analyses, is the maximum principle. For systems, the maximum principle cannot hold due to nonlinear wave interactions. Clearly, rich phenomena await to be discovered for the system of conservation laws with sources. Possible topics include the study of time-periodic solutions, the KAM-type behavior and so forth. We mention here a recent work where the small divisor problem arises in the study of discrete traveling waves ( $\mathrm{Liu}-\mathrm{Yu}[40]$ ). Consider the conservative finite difference scheme for the hyperbolic conservation laws

$$
\frac{u^{n+1}-u^{n}(x)}{\Delta t}=\frac{F\left[u^{n}\right]\left(x+\frac{1}{2} \Delta x\right)-F\left[u^{n}\right]\left(x-\frac{1}{2} \Delta x\right)}{\Delta x},
$$

where $u^{n}(x)$ approximates $u(n \Delta t, x)$ and the functional $F[u]$ satisfies $F[\bar{u}]=$ $f(\bar{u})$ for any constant function $\bar{u}$. We have in mind the basic schemes such as the Lax-Friedrichs and Godunov schemes. It is well-known that discrete 
systems are harder than the corresponding continnum systems. To gain basic understanding, [40] considers the traveling waves for the difference schemes. For the continuous PDEs, the speed $s$ of a wave can always be made zero through a change of independent variables $x$ and $t$. For the discrete systems, however the C-F-L speed $s \Delta t / \Delta x$ is an important variable. If it is rational, then the wave repeats itself after finite steps. For scalar equations, it has been shown that the traveling waves exist and behave similar to the viscous shocks. For systems, there is the striking dependence of the wave shape on the Diophantine property of the C-F-L speed. This is due to the interaction of waves of different characteristic families, which yields the small divisor problem. In [40] it is shown that waves whose speeds are Diophantine exist and are nonlinear stable, but with tail behaviour different from viscous shocks. This singular behaviour is only begun to be understood and consistent with careful numerics. We don't know what happens when the Diophantine property of the wave speed becomes weaker. Deeper understanding requires ideas from discrete dynamics systems.

\section{Multi-Dimensional Gas Flows}

Much of the deep works that have been done for systems of hyperbolic conservation laws are for one space dimension. There are studies of particular solutions for the compressible Euler equations in more than one space dimensions

$$
\begin{aligned}
& \rho_{t}+\sum_{j=1}^{m}\left(\rho v_{j}\right)_{x_{j}}\left(\rho v_{i}\right)+\sum_{j=1}^{m}\left(\rho v_{i} v_{j}\right)_{x_{j}}+p_{x_{i}} \\
& =0, i=1,2, \ldots, m,(\rho E)_{t}+\sum_{j=1}^{m}\left(\rho E v_{j}+p v_{j}\right)_{x_{j}}=0 .
\end{aligned}
$$

The classical book of Courant-Friedrichs [11] is still the good source for these. Self- similar solutions have been constructed; see also Zhang-Zheng [54]. One characteristic of such a solution is that the gas flows around a solid boundary. The solid boundary, the obstacle, has simple geometrical property and defines the flow. The obstacle also helps to stablize the flow and allows for interesting global wave patterns. Examples include spherical waves pushed by an expanding piston and three-dimensional flow around a cone. It would be interesting to study the nonlinear stability of these self-similar flows. For two-dimensional sursonic stationary flows one may view one space dimension as time. The situation is then like one space dimension and methods such as the random choice method can be used for stablity study. 
There are two works on three-dimensional flow around a cone. For straight cone, the flow is self-similar [11]. There is the linearized approach for the study of the flow when the tip of the cone is perturbed (Chen [8]). The Euler equation is singular near the tip and there is delicate analysis of closure in the iterations. The global stability when the cone, away from the tip, is perurbed is done in Lien-Liu [26] using the locally self- similar flows as building blocks in the approximation. The approach is nonlinear and global because one chooses as building blocks the self-similar flows, which represents the far field behaviour of the flow.

There is the local existence of shock propagation based on the linear theory; cf. Majda [44].

To mimic the one-dimensional theory and try to study the general flow without the boundary may not be realistic. In particular, the multi-dimensional Riemann problem is hard, even though the solution is self-similar $u(x, t)=$ $\phi(x / t)$, which reduces the dimension of independent variables by one (LiZhang-Yang [25]). The resulting Euler equations are then of mixed types. Nonlinear equations of mixed types are involved when one tries to resolve the so-called von Neumann paradox on the transition from regular reflection to Mach reflection in the gas flow around the ramp [11]. So far the only analysis is about the algebraic studies of local wave patterns. Fully nonlinear PDE approach is needed for the resolution of the difficult transition problem.

\section{Microscopic Models}

Shock waves are singular, stable occurring in compressible media. It is irreversible. These characteristics make it an interesting and necessary task for study, particularly when the model contains dissipation more general than the viscosity. This is so for the Boltzmann equation and various kinetic models. There are also the particle interacting models, where the dissipation usually comes from the randomness.

One such model is the relaxation model. For a basic study of the simple model of a conservation law and a rate equation

$$
v_{t}+f(v, w)_{x}=0, w_{t}+g(v, w)_{x}=(W(v)-w) / \varepsilon,
$$

see Liu [32]; see also Chen-Levermore-Liu [6] for more general systems. Many kinetic models are of relaxation type $[5,51]$. Basic understandings are gained by comparing the relaxation model with the viscous conservation laws obtained through the Chapman- Enskog expansion ([32,6], Zeng [53]). This approach has the shortcoming that the partial dispersive nature of the models is not considered. It would be nice to study the difference between the relaxation 
model and the associated viscous conservation laws, such as the existence of subshocks in a smooth traveling wave $[32,51]$.

The Boltzmann equation is dissipative as seen from the Chapman-Enskog expansion relating it to the Navier-Stokes equations [5]. However this is so only for large time when waves are sufficiently spreading out. There are works on the Boltzmann shocks, the traveling waves, based on the perturbation from the Navier-Stokes shocks. Such a perturbation method has been successful in dealing with dissipation waves in the time-asymptotic region (Nishida-Imai [45]). For strongly nonlinear soluitons such as the shocks, the method fails to obtain the physical shocks [4]. One needs to come up with a fully nonlinear method for the Boltzmann equation, different from those effective for the viscous conservation laws.

There are progresses in recent years on the macroscopic limits for the interacting particle systems with randomness; cf. Varadhan-Yau [50]. The idea is the sophisticated central limit theorem that works for the situation when the macroscopic solutions, those of the compressible Euler equations, are smooth. It would be interesting to consider the situation when the Euler solutions contain shocks. Again, more nonlinear thinking is needed.

\section{REFERENCES}

1. A. Bressan, A locally contractive metric for systems of conservation laws, Ann. Scuola Norm. Sup. Pisa CI. Sci. (4) 22 (1995), 109-135.

2. A. Bressan, G. Crasta and B. Piccoli, Well posedness of the Cauchy problem for $n \times n$ systems of conservation laws, Mem. Amer. Math. Soc., to appear.

3. A. Bressan and P. LeFloch, Uniqueness of weak solutions to systems of conservation laws, S.I.S.S.A, Trieste, 1996, preprint.

4. R. Caflisch and B. Nicolaenko, Shock profile solutions of the Boltzmann equation, Comm. Math. Phys. 86 (1982), 161-194.

5. C. Cercignani, R. Illn and M. Pulvirenti, The Mathematical Theory of Dilute Gases, Applied Mathematical Science, 106, Springer-Verlag, 1994.

6. G.-Q. Chen, D. Levermore and T.-P. Liu, Hyperbolic conservation laws with stiff relaxation terms and entropy, Comm. Pure Appl. Math. 47 (1994), 787830.

7. G.-Q. Chen and H. Frid, Large-time behaviour of entrpy solutions of conservation laws, J. Differential Equations 152 (1999), 308-357.

8. S. Chen, Non-symmetric conical supersonic flow, in: Hyperbolic Problems: Thoery, Numerics, and Applications, 1 (Zurich 1998), Internat. Ser. Numer. Math. 129, Birkhäuser, Basel, 1999, pp. 149-158. 
9. K.-S. Cheng, Asymptotic behavior of solutions of a conservation law without convexity conditions, J. Differential Equations 40 (1981), 343-376.

10. I.-L. Chern, Local and global interaction for nongenuinely nonlinear hyperbolic conservation laws, Indiana Univ. Math. J., to appear.

11. R. Courant and K.O. Friedrichs, Supersonic Flow and Shock Waves, Springer, 1948.

12. R. DiPerna, Decay and asymptotic behavior of solutions to nonlinear hyperbolic conservation laws, Indiana Univ. Math. J. 24 (1975), 1047-1071.

13. R. DiPerna, Singularities of solutions of nonlinear hyperbolic system of conservation laws, Arch. Rational Mech. Anal. 60 (1975), 75-100.

14. H. Fan and J. Hale, Attractors in inhomogeneous conservation laws and parabolic regularization, Trans. Amer. Math. Soc. 347 (1995), 1239-1254.

15. H. Freistuhler and T.-P. Liu, Nonlinear stability of overcompressive shock waves in a rotatinal invariant system of viscous conservation laws, Comm. Math. Phys. 153 (1993), 147-158.

16. I. Gasser and P. Szmolyan, A geometric singular perturbation analysis of detonation and deflagration waves, SIAM J. Math. Anal. 24 (1993), 968-986.

17. J. Glimm, Solutions in the large for nonlinear hyperbolic systems of equations, Comm. Pure Appl. Math. 18 (1965), 697-715.

18. J. Glimm and P.D. Lax, Decay of solutions of systems of hyperbolic conservation laws, Mem. Amer. Math. Soc. 101 (1970).

19. J. Goodman and Z. Xin, Viscous limits for piecewise smooth solutions to systems of conservation laws, Arch. Rational Mech. Anal. 121 (1992), 235-265.

20. S. Kawashima, Large-time behavior of solutions to hyperbolic-parabolic systems of conservation laws and applications, Proc. Roy. Soc. Edinburgh Sect. A 106 (1987), 169-194.

21. S. Krushkov, First-order quasilinear equations with several space variables, Mat. Sb. 10 (1970), 228-255; English transl.: Math. uSSR Sb. 10 (1970), 217-273.

22. P. D. Lax, C. D. Levermore and S. Venakidis, The generation and propagation of oscillations in dispersive initial value problems and thier limiting behaviour, in: Important Developements in Soliton Theory, Springer Ser. Nonlinear Dynam. Springer, Berlin, 1993, pp. 205-241.

23. P. D. Lax, Hyperbolic systems of conservation laws II, Comm. Pure Appl. Math. 10 (1957), 537-566.

24. P. D. Lax, Hyperbolic System of Conservation Laws and the Mathematical Theory of Shock Waves, Conference Board of the Mathematical Sciences Regional Conference Series in Applied Mathematics, 11, SIAM, Philadelphia, Penn., 1973. 
25. J. Li, T. Zhang and S. Yang, The Two-Dimensional Riemann Problem in Gas Dynamics, Pitman Monograph and Surveys in Pure and Applied Mathematics, Longman, Harlow, 98, 1998.

26. W. Lien and T.-P. Liu, Nonlinear stability of a self-similar 3-dimensional gas flow, Comm. Math. Phys. 204 (1999), 525-549.

27. T.-P. Liu, Riemann problem for general system of hyperbolic conservation laws, J. Differential Equations 18 (1975), 218-234.

28. T.-P. Liu, Pointwise convergence to N-waves for solutions of hyperbolic conservation laws, Bull. Inst. Math. Acad. Sinica 15 (1987), 1-17.

29. T.-P. Liu, Linear and nonlinear large-time behaviors of solutions of hyperbolic conservation laws, Comm. Pure Appl. Math. 30 (1977), 767-796.

30. T.-P. Liu, The deterministic version of the Glimm scheme, Comm. Math. Phys. 57 (1975), 135-148.

31. T.-P. Liu, Admissible solutions of hyperbolic conservation laws, Mem. Amer. Math. Soc. 241 (1981).

32. T.-P. Liu, Hyperbolic conservation laws with relaxation, Comm. Math. Phys. 108 (1987), 153-175.

33. T.-P. Liu, Interactions of nonlinear hyperbolic waves, in: Nonlinear Analysis, F.-C. Liu, T.-P. Liu, eds., World Scientific, Teaneck, NJ, 1991, pp. 171-184.

34. T.-P. Liu, Zero dissipation and stability of shocks, Methods Appl. Anal. 5 (1998), 81-94.

35. T.-P. Liu, Pointwise convergence to shock waves for the system of viscous conservation laws, Comm. Pure Appl. Math. 50 (1997), 1113-1182.

36. T.-P. Liu and J.A. Smoller, On the vacuum state for isentropic gas dynamics equations, Adv. Math. 1 (1980), 345-359.

37. T.-P. Liu and Z. Xin, Pointwise decay to contact discontinuities for systems of viscous consesrvation laws, Asian J. Math. 1 (1997), 34-84.

38. T.-P. Liu and T. Yang, A new entropy functional for a scalar conservation law, Comm. Pure Appl. Math. 52 (1999), 1427-1442.

39. T.-P. Liu and T. Yang, Well-posedness theory for system of conservation laws, Comm. Pure Appl. Math. 52 (1999), 1553-1586.

40. T.-P. Liu and S.-H. Yu, Continuum shock profile for discrete conservation laws, Comm. Pure Appl. Math. 52 (1999), 85-127.

41. T.-P. Liu and S.-H. Yu, Nonlinear stability of weak detonation waves for a combustion model, Comm. Math. Phys. 204 (1999), 551-586.

42. T.-P. Liu and S.-H. Yu, Riemann problem for viscous conservation laws, to appear. 
43. T.-P. Liu and Y. Zeng, Large time behaviour of solutions for general quasilinear hyperbolic-parabolic systems of conservation laws, Mem. Amer. Math. Soc. 599 (1997).

44. A. Majda, Compressible Fluid Flow and Systems of Conservation Laws in Several Space Variables, Springer, New York, 1984.

45. T. Nishida and K. Imai, Global solutions to the initial value problem for the non-linear Boltzmann equation, Publ. Res. Inst. Math. Sci. 12 (1976), 229239.

46. R. Rosales and A. Majda, Weakly nonlinear detonation waves, SIAM J. Appl. Math. 43 (1983), 1086-1118.

47. Y. G. Sinai, Asymptotic behaviour of solutions of 1D-Burgters equation with quasi-periodic forcing, Topol. Methods Nonlinear Anal. 111 (1998), 219-226.

48. J. A. Smoller, Shock Waves and Reaction Diffusion Equations, Springer, New York, 1980.

49. L. Tartar, Compensated compactness and applications to partial differential equations, Res. Notes Math. 39 (1979), 136-210.

50. S.A.S. Varadhan and H.-T. Yau, Diffusive limit of lattice gas with mixing conditions, Asian J. Math. 1 (1997), 623-678.

51. G. B. Whitham, Linear and Nonlinear Waves, John Wiley and Sons, New York, 1974.

52. S.-H. Yu, Zero dissipation limit to solutions with shocks for systems of hyperbolic conservation laws, Arch. Rational Mech. Anal. 146 (1999), 275-370.

53. Y. Zeng, Gas dynamics in thermal nonequilibrium and general hyperbolic systems with relaxation, Arch. Rational Mech. Anal., to appear.

54. T. Zhang and Y. Zheng, Axisymmetric solutions of the Euler equations for polytropic gases, Arch. Rational Mech. Anal. 142 (1998), 253-279.

55. K. Zumbrun and P. Howard, Pointwise semigroup methods and stability of viscous shock waves, Indiana Univ. Math. J. 47 (1998), 741-871.

Department of Mathematics, Stanford University

Stanford, CA 94305, U.S.A.

E-mail: liu@math.stanford.edu 